\title{
Sincronização de Metapopulações em duas Escalas Geográficas $^{1}$
}

V. MANICA 2 , J.A.L. SILVA 3 , Programa de Pós Graduação em Matemática Aplicada, UFRGS - Universidade Federal do Rio Grande do Sul, Av. Bento Gonçalves, 9500, 91509-900 RS, Brasil.

\begin{abstract}
Resumo. Neste trabalho, consideramos um modelo metapopulacional com sítios distribuídos em duas escalas geográficas e analisamos a estabilidade da dinâmica sincronizada. A primeira escala é composta por uma metapopulação com um número arbitrário de sítios, enquanto a segunda escala é composta por um número arbitrário de metapopulações. Durante cada passo de tempo, assumimos que existem 3 processos envolvidos na dinâmica populacional: a) a dinâmica local, que consiste de reprodução e sobrevivência e depende da escolha da função para calcular a densidade de cada sítio; b) a dispersão de indivíduos entre os sítios da primeira escala; e c) a dispersão entre as metapopulações. Analisamos duas maneiras dos sítios sincronizarem, primeiramente consideramos sincronização na escala maior, por conseguinte sincronização nas duas escalas. Para o caso de sincronização nas duas escalas, obtemos um critério para sincronização dependendo de dois parâmetros: o número de Lyapunov e por um parâmetro que depende do processo migratório. No caso da segunda escala estar sincronizada com os respectivos sítios da primeira escala não necessariamente sincronizados, obtemos um critério cujos valores são calculados numericamente.
\end{abstract}

Palavras-chave. Metapopulação, sincronização, números de Lyapunov.

\section{Introdução}

O estudo da sincronização de sistemas dinâmicos populacionais é importante para prever e avaliar o risco de extinção global de espécies. Um fenômeno importante relacionado ao processo de migração é a dinâmica sincronizada. Essa dinâmica corresponde ao caso em que as densidades populacionais em cada sítio evoluem no tempo com mesma amplitude e fase. A importância desse fator reside no fato que se a dinâmica global do sistema não está em sincronia, a população local pode ser recolonizada pelos vizinhos (migrantes) das populações vizinhas ("rescue effect"), isso favorece a persistência da população, conforme Blasius [3]. Por outro lado, o aumento no grau de acoplamento entre as populações favorece a possibilidade de sincronização, podendo tornar a metapopulação vulnerável à extinção, conforme

\footnotetext{
${ }^{1}$ Pesquisa realizada com suporte da CAPES.

${ }^{2}$ vanderlei.manica@ufrgs.br.

${ }_{3}$ jaqx@mat.ufrgs.br.
} 
Earn et al. [6]. Fatores como condições climáticas podem ser suficientes para dar algum tipo de sincronia como, por exemplo, o comportamento de linces canadenses, onde populações em reservas ecológicas geograficamente distantes possuem ciclos populacionais sincronizados [8]. Outro exemplo de sincronização ocorreu no número de casos de dengue na Tailândia, onde uma breve inspeção nos dados coletados entre 1984 e 1996 sugerem sincronia espacial entre as cidades [4].

Estudos analíticos das condições para sincronização caótica consistindo de um número arbitrário de sítios acoplados são feitos em diversos trabalhos da literatura. Allen et al. [1] consideraram um modelo metapopulacional acoplado e, através de simulações numéricas, concluíram que a dinâmica populacional caótica reduz o risco de sincronia. Heino et al. [7] extenderam o modelo proposto por Allen et al. considerando dispersão de indivíduos dependendo da distância entre os sítios e mostraram que esse tipo de assincronia reduz a probabilidade de extinção, concluindo que caos é um caso especial, assim como perturbações locais e outros fatores que causam assincronia. Earn et al. [6] deram sustentação a idéia de que oscilações caóticas reduzem o grau de sincronismo entre os sítios e apresentaram um critério para a estabilidade de oscilações sincronizadas considerando uma metapopulação com um número arbitrário de sítios acoplados. Esses resultados foram generalizados por Silva e Giordani $[9,10]$, que obtiveram um critério para sincronização considerando um mecanismo de migração dependendo da densidade de cada sítio.

Com o objetivo de analisarmos o comportamento de metapopulações, consideramos um modelo metapopulacional com sítios distribuídos em duas escalas, ver Figura 1. Apresentamos resultados sobre a estabilidade transversal assintótica de órbitas sincronizadas e simulações numéricas do modelo considerado.
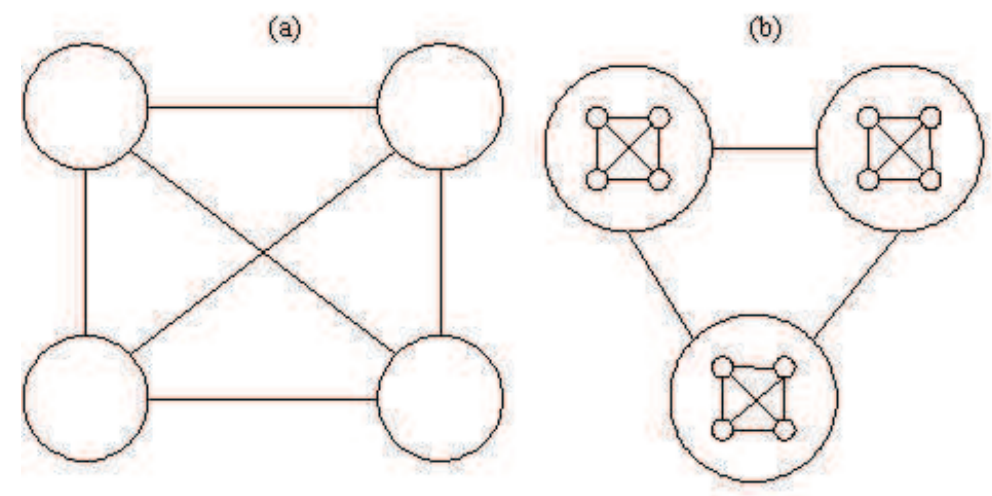

Figura 1: (a) uma metapopulação composta de quatro populações, (b) três metapopulações onde cada metapopulação é composta de quatro populações. 


\section{Modelo Metapopulacional em uma Escala}

O modelo metapopulacional consiste de $N$ sítios enumerados por $1,2, \ldots, N$. Em cada sítio existem indivíduos que estão sujeitos ao processo de dinâmica local e sobrevivência, descrito por uma função $f$ de classe $C^{1}$. Portanto, na falta de migração entre os sítios, a dinâmica local é dada por

$$
x_{t+1}^{i}=f\left(x_{t}^{i}\right), \quad i=1,2, \ldots, N, \quad t=0,1,2, \ldots,
$$

onde $x_{t}^{i}$ representa a densidade de indivíduos no sítio $i$ no passo de tempo $t$. Consideramos que todas as populações são idênticas, no sentido que a dinâmica de cada população é descrita pela mesma função $f$. Dependendo da função $f$, o modelo local dado em (2.1) pode ter um comportamento dinâmico apresentando ciclos estáveis e caos, exemplos de funções podem ser encontrados em [2] e [5].

Após o processo de dinâmica local de cada sítio, uma fração de indivíduos $m$ deixa o sítio $i$ e migra para os sítios vizinhos, onde $0<m<1$. Assim, a densidade de indivíduos que partem do sítio $i$ no passo de tempo $t$ é $m f\left(x_{t}^{i}\right)$. Dos indivíduos que migram dos sítios vizinhos $k$, uma fração $\gamma_{i k}$ chegará para fazer parte da população do sítio $i$ no passo de tempo $t+1$. Consideramos que o processo de migração é 100\% bem sucedido, ou seja, não há perda de indivíduos durante a migração. Assim temos que $\sum_{i=1}^{N} \gamma_{i k}=1$, para todo $k=1, \ldots, N$. Além disso, consideramos que os indivíduos não retornam para o mesmo sítio, ou seja, $\gamma_{k k}=0$ para todo $k=1, \ldots, N$. A matriz $\Gamma=\left[\gamma_{i k}\right]_{i, k=1}^{N}$ é denominada matriz acoplamento entre os sítios da primeira escala, cada termo $\gamma_{i k}$ representa a fração de indivíduos que sai do sítio $k$ no passo de tempo $t$ e passa a fazer parte do sítio $i$ no passo de tempo $t+1$. Fazendo essas considerações, segue que a dinâmica da metapopulação é dada por

$$
x_{t+1}^{i}=(1-m) f\left(x_{t}^{i}\right)+\sum_{\substack{k=1 \\ k \neq i}}^{N} \gamma_{i k} m f\left(x_{t}^{k}\right), \quad i=1,2, \ldots, N .
$$

O primeiro termo do lado direito da equação (2.2) representa os indivíduos que não partiram do sítio $i$ no passo de tempo $t$, enquanto o segundo termo é a soma de todas as contribuições dos sítios vizinhos.

\section{Modelo Metapopulacional em Duas Escalas}

Para distribuição de sítios em duas escalas, consideramos que a primeira escala é formada por uma metapopulação com $N$ sítios, enquanto a segunda escala é formada por metapopulações enumeradas por $1,2, \ldots, n$, formando uma metapopulação com $n N$ sítios. Uma maneira de entendermos o modelo é fazendo uma analogia à dinâmica de indivíduos entre bairros e cidades, a primeira escala seria composta por bairros, enquanto a segunda escala seria composta por cidades. Por simplicidade, vamos nos referir, algumas vezes, aos sítios da primeira escala por bairros e aos sítios da segunda escala por cidades. 
Modelamos a evolução no tempo de um sistema dinâmico de $n N$ equações. Em cada passo de tempo existem três processos considerados: a dinâmica local de cada sítio, a dispersão de indivíduos entre os $N$ sítios da primeira escala e a migração de indivíduos entre os $n$ sítios da segunda escala. Seja $x_{t}^{i j}$ o número de indivíduos no sítio $\{i, j\}$, onde $i$ representa o bairro e $j$ a cidade, para $i=1,2, \ldots, N$, $j=1,2, \ldots, n, t=1,2, \ldots$. Seja $X_{t}^{j}=\left[x_{t}^{1 j}, x_{t}^{2 j}, \ldots, x_{t}^{N j}\right] \in R^{N}$ o vetor populacional da cidade $j$. A dinâmica local de cada metapopulação é descrita por uma função $F: R^{N} \rightarrow R^{N}$ de classe $C^{1}$ que engloba os processos de dinâmica local e migração entre os sítios da primeira escala (bairros). Portanto, na falta de migração entre as metapopulações (cidades), a dinâmica da metapopulação $j$ é dado por

$$
X_{t+1}^{j}=F\left(X_{t}^{j}\right), \quad j=1,2, \ldots, n, \quad t=0,1,2, \ldots
$$

Uma dinâmica apropriada para cada cidade pode ser dada por

$$
F\left(X_{t}^{j}\right)=\left(\begin{array}{c}
(1-m) f\left(x_{t}^{1 j}\right)+\sum_{k=1}^{N} \gamma_{i k} m f\left(x_{t}^{k j}\right) \\
(1-m) f\left(x_{t}^{2 j}\right)+\sum_{k=1}^{N} \gamma_{i k} m f\left(x_{t}^{k j}\right) \\
\vdots \\
(1-m) f\left(x_{t}^{N j}\right)+\sum_{k=1}^{N} \gamma_{i k} m f\left(x_{t}^{k j}\right)
\end{array}\right)
$$

onde $f$ é a função responsável pela dinâmica local de cada bairro, $\gamma_{i k}$ é a taxa de indivíduos que migra do sítio $k$ para o sítio $i$ e $m$ é a fração de migração de indivíduos que migram entre os bairros.

Após o processo de dinâmica local de cada cidade $j$, uma fração de indivíduos $\mu_{i}$ parte de um bairro $i$ e deixa a cidade $j, 0<\mu_{i}<1$, para $i=1, \ldots, N, j=1, \ldots, n$. Dos indivíduos que migram das cidades vizinhas $\ell$, uma proporção $c_{j \ell}$ chegará para fazer parte da população da cidade $j$ no passo de tempo $t+1$. O processo de migração é $100 \%$ bem sucedido, ou seja, não há perda de indivíduos durante a migração, assim temos $\sum_{j=1}^{n} \quad c_{j \ell}=1$. Consideramos também que os indivíduos não retornam para o mesmo sítio, ou seja, $c_{\ell \ell}=0$, para todo $\ell=1, \ldots, n$. A matriz $C=\left[c_{j \ell}\right]_{j, \ell=1}^{n}$ é denominada matriz acoplamento entre os sítios da segunda escala, cada termo $c_{\ell j}$ representa a fração de indivíduos que sai da cidade $j$ no passo de tempo $t$ e passa a fazer parte da cidade $\ell$ no passo de tempo $t+1$ (ver Figura 2).

A densidade de indivíduos que sai do bairro $i$ da cidade $j$ e passa a fazer parte da população da cidade vizinha $\ell$ é dada por $c_{\ell j} \mu_{i} x^{i j}$. Os indivíduos que saem do sítio $\{i, j\}$ e chegam na cidade $\ell$ não necessariamente farão parte do bairro $i$, esses indivíduos se distribuem nos $N$ bairros da cidade $\ell$ numa proporção $\mu_{k i}$, para $k=1, \ldots, N$ (ver Figura 2). Com essas considerações, temos que a densidade de indivíduos que parte do sítio $\ell$ da segunda escala e chega no sítio $\{i, j\}$ da primeira escala é dada por $c_{j \ell} \mu_{i 1} x^{1 \ell}+c_{j \ell} \mu_{i 2} x^{2 \ell}+\ldots+c_{j \ell} \mu_{i N} x^{N \ell}, i=1, \ldots, N, j=1, \ldots, n$. Portanto, a dinâmica de sítios distribuídos em duas escalas é dada por

$$
X_{t+1}^{j}=[I-W] F\left(X_{t}^{j}\right)+\sum_{\substack{\ell=1 \\ \ell \neq j}}^{n} c_{j \ell} \bar{W} F\left(X_{t}^{\ell}\right), \quad j=1, \ldots, n,
$$

onde $I$ é a matriz identidade, $W=\operatorname{diag}\left(\mu_{1}, \mu_{2}, \ldots, \mu_{N}\right)$ e a matriz $\bar{W}$ é dada por 


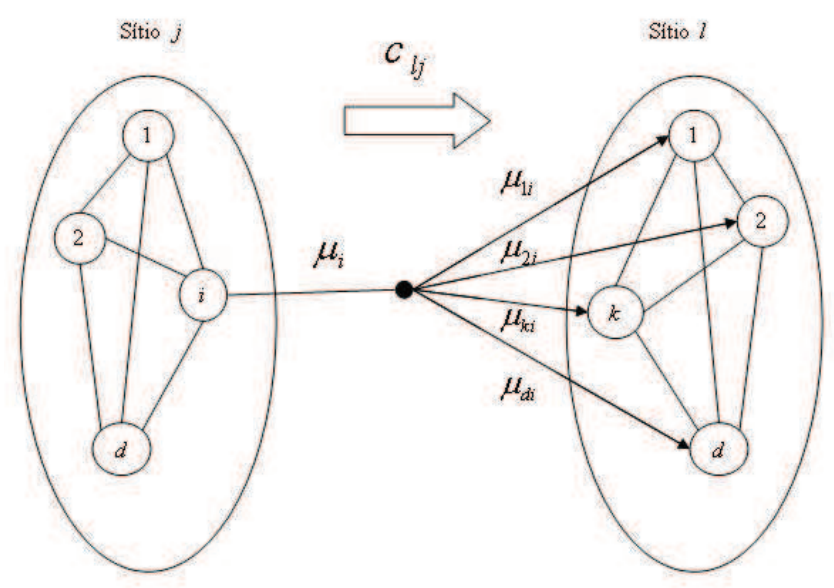

Figura 2: Indivíduos migram do sítio $j$ para o sítio $\ell$ numa proporção $c_{\ell j}$. Os indivíduos que chegam em $\ell$ saindo do sítio $\{i, j\}$, com fração de migração $\mu_{i}$, se distribuem no sítio vizinho numa proporção $\mu_{k i}, k=1, \ldots, N$.

$$
\bar{W}=\left[\begin{array}{cccc}
\mu_{11} & \mu_{12} & \ldots & \mu_{1 N} \\
\mu_{21} & \mu_{22} & \ddots & \vdots \\
\vdots & \ddots & \ddots & \mu_{N-1 N} \\
\mu_{N 1} & \ldots & \mu_{N N-1} & \mu_{N N}
\end{array}\right]
$$

Obtendo-se assim, um sistema dinâmico de $n N$ equações. O primeiro termo do lado direito da equação (3.3) representa os indivíduos que não partiram da metapopulação $j$ no passo de tempo $t$, enquanto o segundo termo conta as contribuições das metapopulações vizinhas.

\section{Sincronização e Estabilidade Transversal}

Consideraremos que a sincronização pode ocorrer de dois modos diferentes, são eles: sincronização somente na escala maior e sincronização nas duas escalas. No primeiro caso, todas as cidades estão sincronizadas com os respectivos bairros não necessariamente sincronizados. Neste caso, ocorre sincronização se $X_{t}^{j}=\mathbf{X}_{t}$, onde $\mathbf{X}_{t}=\left(x_{t}^{1}, x_{t}^{2}, \ldots, x_{t}^{N}\right) \in R^{N}$, para todo $j=1, \ldots, n$. Já no seggundo caso, todas as cidades estão sincronizadas com os respectivos bairros sincronizados. Neste caso, ocorre sincronização se $X_{t}^{j}=\mathbf{X}_{t}$, onde $\mathbf{X}_{t}=\left(x_{t}, x_{t}, \ldots, x_{t}\right) \in R^{N}$, para todo $j=1, \ldots, n$.

Assim, considerando que a densidade dos sítios na escala maior é a mesma, $X_{t}^{j}=\left(x_{t}^{1}, x_{t}^{2}, \ldots, x_{t}^{N}\right)$, para todo $j=1,2, \ldots, n$, e substituindo em (3.3), temos que 


$$
\mathbf{X}_{t+1}=[I-W] F\left(\mathbf{X}_{t}\right)+\sum_{\ell=1}^{n} c_{j \ell} \bar{W} F\left(\mathbf{X}_{t}\right), \quad j=1, \ldots, n
$$

Supomos que $\sum_{\ell=1}^{n} c_{j \ell}=1$, para todo $j=1, \ldots, n$, temos que

$$
\mathbf{X}_{t+1}=(I-W+\bar{W}) F\left(\mathbf{X}_{t}\right)
$$

Portanto, a dinâmica metapopulacional no estado sincronizado satisfaz (4.1). Nosso interesse é estudar a estabilidade assintótica do sistema (3.3), isto é, quando órbitas populacionais que iniciam próximas ao estado sincronizado serão atraídas para este estado. Para fazer esta análise, usaremos o teorema a seguir que é fundamental no processo de linearização.

Teorema 4.1. Seja $F: R^{N} \longrightarrow R^{N}$ uma função de classe $C^{1}$. Seja $C$ matriz acoplamento entre os sítios e diagonalizável, $S_{t}=\left(\mathbf{X}_{t}, \mathbf{X}_{t}, \ldots, \mathbf{X}_{t}\right) \in R^{n \times N}$ o estado sincronizado da metapopulação a cada passo de tempo, onde $\mathbf{X}_{t}=\left(x_{t}^{1}, x_{t}^{2}, \ldots, x_{t}^{N}\right) \in$ $R^{N}$. Então o sistema linear associado ao sistema não linear (3.3) é dado por

$$
Y_{t+1}=\bigoplus_{j=1}^{n}\left[I-W+\lambda_{j} \bar{W}\right] D F\left(\mathbf{X}_{t}\right) Y_{t}
$$

onde $\lambda_{j}$ são os autovalores da matriz $C, Y_{t}$ é uma mudança de variáveis, $D F\left(\boldsymbol{X}_{t}\right)$ é a matriz Jacobiana do modelo metapopulacinal dado em (2.2) e $\bigoplus$ representa o desacoplamento por blocos de matrizes, isto é,

$$
\bigoplus_{j=1}^{n} A_{j}=\left(\begin{array}{cccc}
A_{1} & & & \\
& A_{2} & & \\
& & \ddots & \\
& & & A_{n}
\end{array}\right)
$$

Demonstração. Linearizando o sistema (3.3) em torno do estado sincronizado $S_{t}$, obtemos a seguinte equação para a evolução da perturbação $\Delta_{t}$,

$$
\Delta_{t+1}=J\left(S_{t}\right) \Delta_{t}
$$

onde $\Delta_{t}$ é a perturbação transversal e $J\left(S_{t}\right)$ é a matriz Jacobiana $n N \times n N$ do sistema (3.3) aplicada no estado sincronizado.

Pela definição de produto de Kronecker ${ }^{4}$, a matriz Jacobiana pode ser escrita na forma

$$
J\left(S_{t}\right)=I \bigotimes D F\left(\mathbf{x}_{t}\right)-I \bigotimes W D F\left(\mathbf{x}_{t}\right)+C \bigotimes \bar{W} D F\left(\mathbf{x}_{t}\right)
$$

A matriz $C$ é diagonalizável, então existe $Q$ matriz não singular que diagonaliza $C$, isto é, $Q C Q^{-1}=\Lambda^{*}$, onde $\Lambda^{*}=\operatorname{diag}\left(\lambda_{0}, \lambda_{1}, \ldots, \lambda_{n-1}\right)$.

\footnotetext{
${ }^{4}$ Seja $A=\left[a_{i, j}\right]_{i, j=1}^{m} \in R^{m \times m}$ e $B=\left[b_{i, j}\right]_{i, j=1}^{n} \in R^{n \times n}$, o produto de Kronecker é definido por $A \otimes B=\left[a_{i, j} B\right]_{i, j=1}^{n} \in R^{m n \times m n}$.
} 
Considerando a mudança de variáveis, $Y_{t}=(Q \otimes I) \Delta_{t}$, temos que $Y_{t+1}=(Q \otimes I) \Delta_{t+1}$

$Y_{t+1}=(Q \otimes I)\left(I \otimes D F\left(\mathbf{X}_{t}\right)-I \otimes W D F\left(\mathbf{X}_{t}\right)+C \otimes \bar{W} D F\left(\mathbf{X}_{t}\right)\right) \Delta_{t}$, $Y_{t+1}=\left(Q \otimes D F\left(\mathbf{X}_{t}\right)-Q \otimes W D F\left(\mathbf{X}_{t}\right)+Q C \otimes \bar{W} D F\left(\mathbf{X}_{t}\right)\right) \Delta_{t}$, utilizando propriedades do produto de Kronecker.

Pelo fato de $Y_{t}=(Q \otimes I) \Delta_{t}$, temos que $\Delta_{t}=(Q \otimes I)^{-1} Y_{t}$, logo $Y_{t+1}=\left(Q \otimes D F\left(\mathbf{X}_{t}\right)-Q \otimes W D F\left(\mathbf{X}_{t}\right)+Q C \otimes \bar{W} D F\left(\mathbf{X}_{t}\right)\right)(Q \otimes I)^{-1} Y_{t}$, $Y_{t+1}=\left(Q \otimes D F\left(\mathbf{X}_{t}\right)-Q \otimes W D F\left(\mathbf{X}_{t}\right)+Q C \otimes \bar{W} D F\left(\mathbf{X}_{t}\right)\right)\left(Q^{-1} \otimes I\right) Y_{t}$, $Y_{t+1}=\left(I \otimes D F\left(\mathbf{X}_{t}\right)-I \otimes W D F\left(\mathbf{X}_{t}\right)+\Lambda^{*} \otimes \bar{W} D F\left(\mathbf{X}_{t}\right)\right) Y_{t}$, obtendo-se o desacoplamento por blocos de matrizes.

A importância desse teorema reside no fato que a estabilidade do sistema não linear pode ser avaliada através do sistema linearizado. Mais precisamente, denotando por $r$ o raio espectral da matriz $\bigoplus_{j=1}^{n-1}\left[I-W+\lambda_{j} \bar{W}\right] D F\left(\mathbf{X}_{t}\right)$, o ponto de equilíbrio é assintoticamente estável se $r<1$, e instável se $r>1$. O desacoplamento nos permite determinar os números de Lyapunov de $J\left(S_{t}\right)$ a partir das $n$ matrizes $\left[I-W+\lambda_{j} \bar{W}\right] D F\left(\mathbf{X}_{t}\right)$ de ordem $N \times N$. É importante observar que $\lambda_{0}=1$ é um autovalor da matriz de configuração $C$ que corresponde aos números de Lyapunov da solução sincronizada. Assim, a estabilidade assintótica pode ser avaliada através dos $(n-1)$ blocos restantes, ou seja, através dos números transversais de Lyapunov.

Considerando a linearização do sistema (3.3) e o teorema 4.1, queremos que a perturbação $\Delta_{t}$ tenda a zero ao $t \rightarrow \infty$. Isto ocorrerá se e somente se

$$
K\left(\mathbf{X}_{0}\right)=\max _{j=1, \ldots, n-1}\left(\lim _{\tau \rightarrow \infty}\left\|P_{\tau-1} \cdot \ldots \cdot P_{1} P_{0}\right\|^{1 / \tau}\right)<1,
$$

onde $P_{t}=\left(I-W+\lambda_{j} \bar{W}\right) D F\left(\mathbf{X}_{t}\right)$ e $K\left(\mathbf{X}_{0}\right)$ é o maior número transversal de Lyapunov que depende da condição inicial $\mathbf{X}_{0}$. A seguir, analisaremos a evolução da perturbação transversal para os dois modos de sincronização:

1. Sincronização na escala maior: a matriz Jacobiana $D F\left(\mathbf{X}_{t}\right)$ do sistema metapopulacional (2.2), calculada em $\mathbf{X}_{t}=\left(x_{t}^{1}, x_{t}^{2}, \ldots, x_{t}^{N}\right)$, possui suas entradas dadas por

$$
\alpha_{k i}= \begin{cases}(1-m) f^{\prime}\left(x_{t}^{i}\right), & \text { se } \mathrm{k}=\mathrm{i} \\ \gamma_{k i} m f^{\prime}\left(x_{t}^{i}\right), & \text { se } \mathrm{k} \neq \mathrm{i} .\end{cases}
$$

Denotando por $\Omega_{s m}$ o atrator sincronizado para o sistema metapopulacional em duas escalas e considerando hipóteses adequadas de integrabilidade sobre $F$, podemos tirar a dependência da condição inicial e estabelecer uma condição suficiente para a estabilidade assintótica de $\Omega_{s m}$, dado pelo teorema a seguir.

Teorema 4.2. O maior número transversal de Lyapunov do atrator $\Omega_{s m}$ é dado por (4.4). Além disso, se

$$
K<1,
$$

o atrator $\Omega_{s m}$ é transversalmente assintoticamente estável.

Para o caso $K<1$ ocorre sincronização na escala maior, enquanto para $K>1$ não ocorre esse tipo de sincronização. Os valores de $K$ são calculados numericamente 
utilizando-se um algoritmo descrito no livro de Aligood [2] que calcula a expansão de órbitas em $N$ direções ortonormais.

2. Sincronização nas duas escalas: a matriz Jacobiana $D F\left(\mathbf{X}_{t}\right)$ de $(2.2)$, calculada em $\mathbf{X}_{t}=\left(x_{t}, x_{t}, \ldots, x_{t}\right) \in R^{N}$, possui suas entradas dadas por

$$
\alpha_{k i}= \begin{cases}(1-m) f^{\prime}\left(x_{t}\right), & \text { se } \mathrm{k}=\mathrm{i} \\ \gamma_{k i} m f^{\prime}\left(x_{t}\right), & \text { se } \mathrm{k} \neq \mathrm{i}\end{cases}
$$

que pode ser escrita como

$$
D F\left(\mathbf{X}_{t}\right)=(I-m B) f^{\prime}\left(x_{t}\right)
$$

onde $B=I-\Gamma, \Gamma$ é a matriz de acoplamento entre os sítios da primeira escala.

Portanto, $P_{t}=\left(I-W+\lambda_{j} \bar{W}\right)(I-m B) f^{\prime}\left(x_{t}\right)$, para cada $\lambda_{j}, j=1, \ldots, n-1$. Dessa forma,

$$
\begin{aligned}
& \left\|P_{\tau-1} \cdot \ldots \cdot P_{1} P_{0}\right\| \\
& =\left\|\left(I-W+\lambda_{j} \bar{W}\right)(I-m B) f^{\prime}\left(x_{\tau-1}\right) \cdot \ldots \cdot\left(I-W+\lambda_{j} \bar{W}\right)(I-m B) f^{\prime}\left(x_{0}\right)\right\| \\
& =\left(\prod_{t=0}^{\tau-1}\left|f^{\prime}\left(x_{t}\right)\right|\right)\left\|\left(I-W+\lambda_{j} \bar{W}\right)(I-m B) \cdot \ldots \cdot\left(I-W+\lambda_{j} \bar{W}\right)(I-m B)\right\| \\
& =\left(\prod_{t=0}^{\tau-1}\left|f^{\prime}\left(x_{t}\right)\right|\right)\left\|\left(\left(I-W+\lambda_{j} \bar{W}\right)(I-m B)\right)^{\tau}\right\| . \\
& \quad \operatorname{Assim}, \lim _{\tau \rightarrow \infty}\left\|P_{\tau-1} \cdot \ldots \cdot P_{1} P_{0}\right\|^{1 / \tau}=L\left(x_{0}\right) \Lambda^{j},
\end{aligned}
$$

onde $L\left(x_{0}\right)=\lim _{\tau \rightarrow \infty}\left(\prod_{t=0}^{\tau-1}\left|f^{\prime}\left(x_{t}\right)\right|\right)^{1 / \tau}$ é o número de Lyapunov começando em $x_{0}$ e $\Lambda^{j}$ é o raio espectral de $\left(I-W+\lambda_{j} \bar{W}\right)(I-m B)$, para cada $\lambda_{j}, j=1, \ldots, n-1$.

Considerando hipóteses adequadas de integrabilidade sobre $f$, podemos eliminar a dependência do número de Lyapunov de $x_{0}$ e estabelecer uma condição suficiente para a estabilidade assintótica,

$$
K=L \Lambda<1,
$$

onde $\Lambda=\max _{j=1, \ldots, n-1}\left(\Lambda^{j}\right)$.

Na região onde o parâmetro $L \Lambda<1$ ocorre sincronização nas duas escalas, enquanto para $L \Lambda>1$ não ocorre esse tipo de sincronização. O valor $L$ depende apenas da dinâmica local do sítio, enquanto $\Lambda$ depende do processo migratório entre os sítios de ambas as escalas. Na próxima seção, apresenta-se simulações numéricas do modelo metapopulacional em duas escalas.

\section{Simulações Numéricas}

Considera-se que a dinâmica local de cada sítio é governada pela função exponencial logística que é dada por

$$
f(x)=x \exp (r(1-x))
$$


onde $r$ representa a taxa de crescimento populacional e $x$ a densidade populacional. Para um sítio isolado, a dinâmica dada por (5.1) apresenta ciclos estáveis e caos [1].

Para avaliarmos o comportamento do modelo metapopulacional em duas escalas, calcula-se o erro de sincronização que é dado por

$$
e_{t}=\frac{1}{n N} \sum_{i=1}^{N} \sum_{j=1}^{n}\left|x_{t}^{i, j}-x_{t}^{i, j+1}\right|
$$

onde $x_{t}^{N+1, j}=x_{t}^{1, j}, x_{t}^{i, n+1}=x_{t}^{i, 1}$. Assim, obtemos sincronização entre as cidades se $e_{t} \rightarrow 0$ quando $t \rightarrow \infty$.

Na Figura 3, plota-se o maior número transvesal de Lyapunov em função do parâmetro $\mu$. Observe que para plotarmos o erro de sincronização, analisamos a evolução do sistema dinâmico dado em (3.3), enquanto os números de Lyapunov são calculados em (4.4). Considera-se o modelo com 5 cidades e 5 bairros. A dinâmica de cada bairro é dada pela função exponencial logística com $r=3,1$, cuja dinâmica local é caótica e o número de Lyapunov é aproximadamente $L=1,3276$. As densidades populacionais iniciais de cada sítio são escolhidas aleatoriamente próximas ao estado sincronizado.

Consideramos que ambas as escalas estão acopladas com os dois vizinhos mais próximos em forma de anel. Os autovalores da matriz de configuração $C$ são dados por: $\lambda_{0}=1, \lambda_{1}=-0,809$ com multiplicidade $2, \lambda_{2}=0,309$ com multiplicidade 2 .

Ao variarmos o parâmetro $\mu$, consideramos que a matriz de configuração $W$ é dada por $W=\operatorname{diag}(\mu, \mu, \mu, \mu, \mu)$. Ao ocorrer o terceiro processo (migração entre as cidades), os indivíduos se distribuem uniformemente nos sítios da cidade vizinha. Assim, temos que

$$
\begin{aligned}
& \left(I-W+\lambda_{j} \bar{W}\right)= \\
& \left(\begin{array}{ccccc}
1-\mu+\frac{\lambda_{j} \mu}{5} & \frac{\lambda_{j} \mu}{5} & \frac{\lambda_{j} \mu}{5} & \frac{\lambda_{j} \mu}{5^{5}} & \frac{\lambda_{j} \mu}{5} \\
\frac{\lambda_{j} \mu}{5} & 1-\mu+\frac{\lambda_{j} \mu}{5} & \frac{\lambda_{j} \mu}{5} & \frac{\lambda_{j} \mu}{5} & \frac{\lambda_{j} \mu}{5} \\
\frac{\lambda_{j} \mu}{5} & \frac{\lambda_{j} \mu}{5} & 1-\mu+\frac{\lambda_{j} \mu}{5} & \frac{\lambda_{j} \mu}{5} & \frac{\lambda_{j} \mu}{5} \\
\frac{\lambda_{j} \mu}{5} & \frac{\lambda_{j} \mu}{5} & \frac{\lambda_{j} \mu}{5} & 1-\mu+\frac{\lambda_{j} \mu}{5} & \frac{\lambda_{j} \mu}{5} \\
\frac{\lambda_{j} \mu}{5} & \frac{\lambda_{j} \mu}{5} & \frac{\lambda_{j} \mu}{5} & \frac{\lambda_{j} \mu}{5} & 1-\mu+\frac{\lambda_{j} \mu}{5}
\end{array}\right)
\end{aligned}
$$

Portanto, para plotarmos os números tranversais de Lyapunov, calcula-se a evolução das matrizes $P_{t}=\left(I-W+\lambda_{j} \bar{W}\right) D F\left(\mathbf{X}_{t}\right)$ em $N$ direções ortonormais, onde $D F\left(\mathbf{X}_{t}\right)$ é a matriz Jacobiana de (2.2), e plota-se o maior valor que indica se as órbitas do sistema metapopulacional estão expandindo $(K>1)$ ou contraindo $(K<1)$ nas direções transversais ao atrator metapopulacional sincronizado.

Com as considerações feitas na dinâmica metapopulacional, para o caso de $m=$ 0,02, observa-se os seguintes comportamentos nos intervalos aproximados: $\mu \in$ $(0,0,02]$, não ocorre sincronização; $\mu \in(0,02,0,29]$, ocorre sincronização entre as cidades; $\mu \in(0,29,0,47]$, cidades não sincronizam, mas os bairros de cada cidade sincronizam; $\mu \in(0,47,0,98]$, ocorre sincronização entre as cidades e os bairros; $\mu \in(0,98,1)$, não ocorre sincronização (ver figura $3(\mathrm{a})$ ). O aumento na taxa de migração entre os bairros para $m=0,1$ faz as cidades sincronizarem com taxas de migração próximas a zero (ver figura 3(c)). Nesse caso, observa-se os seguintes comportamentos nos intervalos aproximados: $\mu \in(0,0,24]$, ocorre sincronização 
entre as cidades; $\mu \in(0,24,0,47]$, as cidades não sincronizam, mas os bairros de cada cidade sincronizam; $\mu \in(0,47,0,98]$, ocorre sincronização entre as cidades e os bairros; $\mu \in(0,98,1)$, não ocorre sincronização.
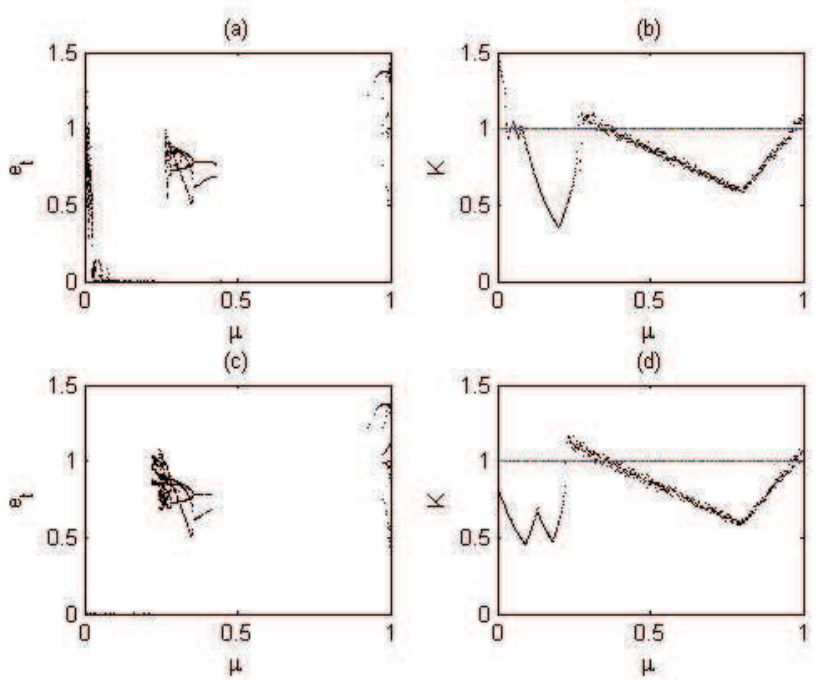

Figura 3: Erro de sincronização ((a) e (c)) com o respectivo (e maior) número transversal de Lyapunov ((b) e (d)) em função de $\mu$, considerando sincronização na escala maior. Os sítios estão acoplados em forma de anel com os dois vizinhos mais próximos, $r=3,1, n=5, d=5$. (a) $m=0,02$, (c) $m=0,1$.

Na Figura 4, apresenta-se os gráficos sítios-tempo, após o descarte de transientes. Os sítios estão ao longo do eixo vertical e são numerados de 1 a $n N$, onde as $N$ primeiras células correspondem à cidade 1 , as $\mathrm{N}$ seguintes células à cidade 2 e assim por diante. As células são pintadas em seis cores de acordo com a densidade de cada sítio, para densidades altas as células são pintadas de branca e para densidades baixas as células são pintadas de preta, enquanto para densidades intermediárias as células são pintadas de tons cinzas. As condições do modelo metapopulacional são as mesmas descritas para plotar a Figura 3(a), mas com $\mu$ fixo. Em (a) $\mu=0,01$, o maior número transversal de Lyapunov é maior que 1 e não ocorre sincronização. Em (b) $\mu=0,2$, o maior número transversal é inferior a 1 e ocorre sincronização entre as cidades, além disso, essa sincronização possui um ciclo de período 2 . Em (c) $\mu=0,3$, não ocorre sincronização entre as cidades, mas ocorre sincronização entre os bairros de cada cidade. Em (d) $\mu=0,5$ e ocorre sincronização nas duas escalas. Para observarmos que ocorreu sincronização entre as cidades, devemos olhar para as células no eixo vertical de 5 em 5 pois cada cidade possui 5 bairros. 


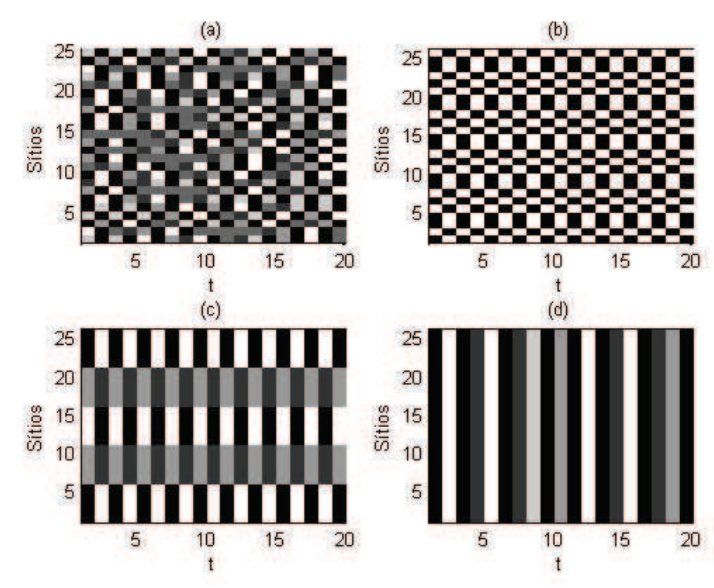

Figura 4: Gráficos sítios-tempo. Mesmas considerações para plotar a figura 3(a), com $\mu$ fixo. Em (a) $\mu=0,01$, não ocorre sincronização; (b) $\mu=0,2$, ocorre sincronização entre as cidades; (c) $\mu=0,3$, não ocorre sincronização entre as cidades, mas os bairros sincronizam; (d) $\mu=0,5$, ocorre sincronização nas duas escalas.

\section{Conclusão}

Sincronização de sistemas metapopulacionais está fortemente relacionada com a extinção de populações, dessa forma, resultados de vários estudos podem levantar informações úteis para evitá-la. Neste trabalho, um modelo metapopulacional de sítios distribuídos em duas escalas conectados via processo de migração é proposto com o objetivo de estudarmos a estabilidade assintótica da solução sincronizada. Essa análise é feita através do processo de linearização e calcula-se numericamente os números de Lyapunov, obtendo-se condições suficientes para a sincronização entre metapopulações. Essas condições são obtidas a partir da decomposição por blocos da matriz Jacobiana. O ponto chave é o fato de um dos blocos corresponder a matriz variacional das equações do atrator metapopulacional, enquanto os demais blocos correspondem às direções transversais e ditam sua estabilidade. Essa técnica nos traz informações sobre o comportamento dos atratores sincronizados para metapopulações e de sua estabilidade local. Um exemplo numérico considerando a dinâmica local dada pela função exponencial logística e distribuição uniforme entre as cidades mostra os diferentes padrões metapopulacionais ao variarmos as taxas de migração de ambas as escalas. Entretanto, mais trabalho precisa ser feito para descrever e testar as diferentes hipóteses que podem ser feitas sobre o modelo para descrever esses padrões.

\footnotetext{
Abstract. In this paper, a metapopulation model composed of patches distributed in two geographic scales is proposed in order to study the stability of the synchronous dynamics. The first scale is a metapopulation consisting of equal patches while the second scale is composed of an arbitrary number of metapopulations. During each time step, we assume that there are three processes involved in the population
} 
dynamics: a) the local dynamics, which consists of reproduction and survival and depends only on function chosen to calculate the density of each patch before migration; b) the dispersal of individuals between the patches of the first scale; and c) the movement between the metapopulations. We examine two kinds of synchronizations: when each patch has the same density (both scales are synchronized), and when each metapopulation has the same density patches with its patches not necessary synchronized, that is, only the second scale is synchronized. A stability criterion is obtained based on the computation of the transversal Lyapunov number of attractors on the synchronous invariant manifold. For case where both scales are synchronized, the criterion is determined by two terms: the Lyapunov number that depends only on the local dynamics of each isolated patch and a term that depends on the migration process. For the case where only the second scale is synchronized, the transversal Lyapunov number is calculated numerically.

Keywords. Metapopulation, synchronization, Lyapunov number.

\section{Referências}

[1] J.C. Allen, W.M. Schauffer, D. Rosko, Chaos reduces species extinction by amplifying local population noise, Nature 364 (1993), 229-232.

[2] K.T. Alligood, T.D. Sauer, J.A. Yorke, "CHAOS An introduction to Dynamical Systems", Springer-Verlag, New York, 1997.

[3] B. Blasius, A. Huppert, L. Stone, Complex dynamics and phase synchronization in saptially extended ecological systems, Nature 399 (1999), 353-359.

[4] D.A.T. Cummings, R.A. Irizarry, N.E. Huang, T.P. Endy, A. Nisalak, K. Ungchusak, D.S. Burke, Travelling waves in the occurrence of dengue haemorrhogic fever in Thailand, Nature 427 (2004), 344-347.

[5] L.J. Díaz, D.R. Jorge, "Uma Introdução aos Sistemas Dinâmicos via Frações Contínuas", IMPA, 2007.

[6] D.J., Earn, S.A. Levin, P. Rohani, Coherence and Conservation, Science 290 (2000), 1360-1364.

[7] M. Heino, V. Kaitala, E. Ranta, J. Lindström, Synchronous dynamics and rates of extinction in spatially structured populations, Proc. R. Soc. London B $\mathbf{2 6 4}$ (1997) 481-486.

[8] R.M. May, A.L. Lloyd, Synchronicity, chaos and population cycles: spatial coherence in an uncertain world, Trends Ecol. Evol., 14 (1999), 417-418.

[9] J.A.L. Silva, J.A. Barrionuevo, F.T. Giordani, Synchronism in population networks with non linear coupling, Nonlinear Analysis: Real World Applications, 11 (2010), 1005-1016.

[10] J.A.L. Silva, F.T. Giordani, Density-dependent migration and synchronism in metapopulations, Bull. Math. Biol., 68 (2006), 451-465. 